\title{
LA RELACIÓN COSTO-CALIDAD: ALGUNOS ASPECTOS ECONÓMICOS DEL CONFORT TÉRMICO
}

\author{
(THE COST-QUALITY RELATION: SOME ECONOMIC ASPECTS OF THERMAL \\ COMFORT)
}

\author{
Beatriz Cecilia Amarilla, arquitecto investigador \\ Comisión de Investigaciones Científicas de la Provincia de Buenos Aires. \\ Laboratorio de Investigaciones del Territorio y el Ambiente. (CIC/LINTA) - La Plata, \\ REPÚBLICA ARGENTINA
}

Fecha de recepción: 24 - II - 93

\begin{abstract}
RESUMEN
El presente trabajo propone una metodología para analizar conjuntamente aspectos de costo y calidad en los edificios para vivienda. A los efectos de ilustrar mediante un ejemplo de aplicación de dicha metodología, el "costo" se ha referido a la construcción, mantenimiento y energía para calefacción; la "calidad", por su parte, se ha identificado con algunos aspectos del confort térmico.
\end{abstract}

Se intenta demostrar que no siempre existe una relación directamente proporcional entre el costo de los edificios y las calidades objetivas que en ellos se alcanzan.

Se estudiaron y relacionaron variables de tipo bioclimático, social, económico, tecnológico y morfológico. El objetivo final es obtener un "costo ponderado" (costo en relación al beneficio térmico conseguido). Ello permite determinar un orden de prioridades entre varias soluciones altemativas que se proponen para la construcción masiva de viviendas.

\section{SUMMARY}

This paper proposes a methodology for joint analysis of cost and quality in dwelling buildings. In order to give an illustrative example of the application of this methodology, the "cost" referred to the construction, maintenance and energy for heating, while the "quality", in its turn, referred to some aspects of thermal comfort.

The authors try to prove that there is not always a directly proportional relation between the cost of buildings and the real quality reached in them.

Bioclimatic, social, economic, technological and morphological variables have been analyzed. The final objective is to obtain a "weighting cost" (cost according to the achieved thermal benefit). This allows the determination of an order of priorities among various alternative solutions proposed for mass construction of dwellings.

\section{INTRODUCCIÓN: EL COSTO Y LA CALIDAD}

La carencia de viviendas plantea en la mayoría de los países de Iberoamérica un bien conocido problema. En la República Argentina, el déficit habitacional por precariedad o hacinamiento supera los dos millones y medio de unidades. Una gran parte de ese déficit se concentra en el conurbano bonaerense, es decir, las áreas próximas a la ciudad de Buenos Aires. (Fig. 1).
La puesta en marcha de cualquier política habitacional se enfrenta siempre a un doble problema: el del costo y el de la calidad de las viviendas, de su equipamiento, y de las redes de la infraestructura urbana. Los resultados alcanzables serán siempre deficientes si el problema se plantea exclusivamente como una ecuación de costos. En este caso, los recursos económicos limitados que el Estado puede destinar, y la escala masiva que presenta el déficit, llevan inexorablemente a la construcción de viviendas de baja calidad y de reducida vida útil. 


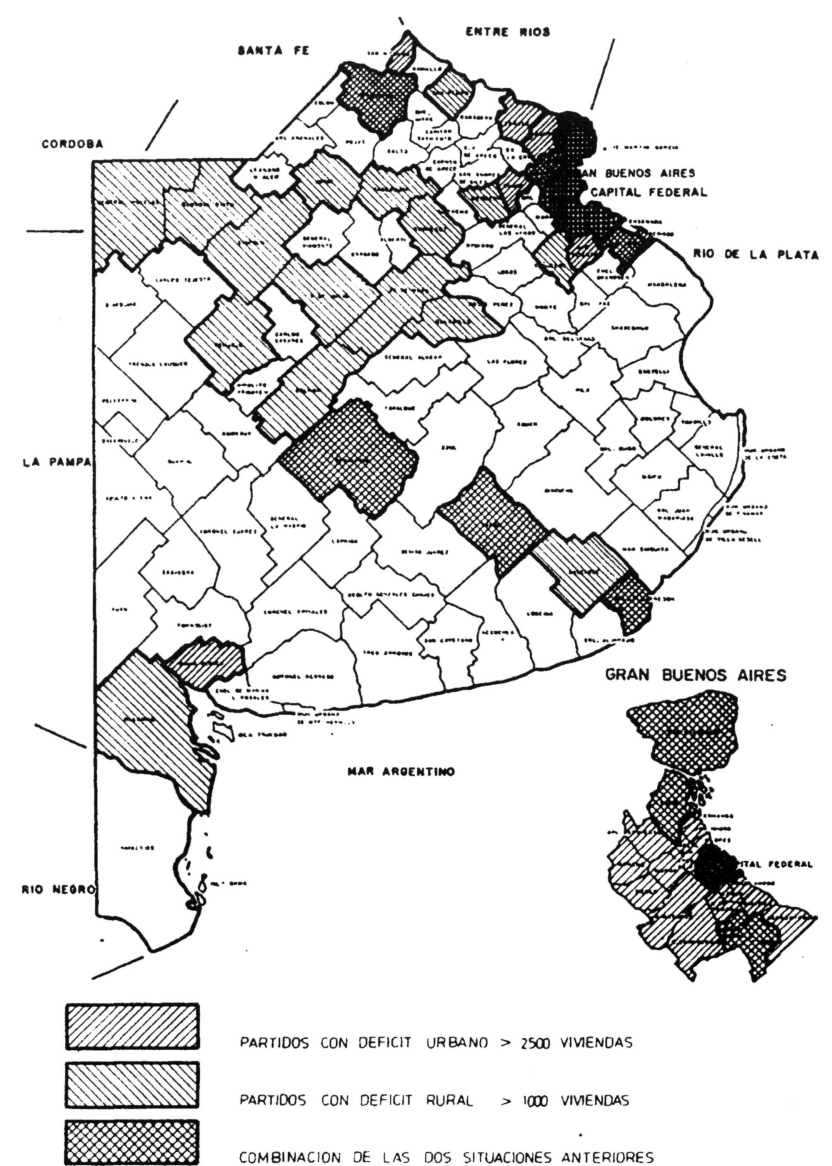

Fig. 1.- Partidos con mayor deficit habitacional (urbano, rural y total).

El problema del déficit habitacional debe resolverse en términos de la relación costo-calidad. El objetivo final debe ser optimizar la calidad de vida de los usuarios, teniendo en cuenta su entorno geográfico, bioclimático, económico y sociocultural.

El presente trabajo intenta demostrar que no existe una relación directamente proporcional entre el costo de construcción y la calidad del producto terminado. Y que es posible obtener un buen rendimiento de la operación económica con desembolsos moderados de capital.

Uno de los problemas clave que impide poner en práctica los conceptos anteriores en Iberoamérica es la falta de material estadístico, o la dispersión de la información existente. No hay, en general, bancos de datos fácilmente accesibles para los proyectistas, durante el proceso de diseño de los edificios. Lo ideal sería contar con datos sobre alternativas de diseño -diferentes tecnologías, materiales, morfología de los cerramientos, etc.- que permitieran, a nivel del anteproyecto, probar distintas soluciones de costo y calidad hasta encontrar la combinación óptima.
La falta de datos conduce a situaciones con frecuencia indeseables: el uso de pautas de diseño no objetivas, el manejo de datos cuantitativos poco precisos; o la necesidad de recurrir a cálculos extensos y complejos, que perturban el proceso global de diseño, y que suelen abandonarse por esos mismos motivos.

La calidad en arquitectura es un concepto complejo: abarca y combina una serie muy extensa de atributos parciales. Algunos de ellos pueden medirse en unidades determinadas, igual que los costos (por ejemplo, la transmisión térmica, el aislamiento acústico, el nivel de iluminación); otras son de carácter esencialmente subjetivo, y escapan a cualquier criterio de evaluación cuantitativa.

El análisis que se presenta es una síntesis de un trabajo de investigación, perteneciente a una serie dedicada al estudio de la relación costo-calidad en la construcción de viviendas ${ }^{(1)}$. En la primera parte, se hace un resumen de los parámetros de evaluación considerados y de los factores que los definen y caracterizan. La segunda parte está dedicada a la aplicación de algunos conceptos metodológicos que permiten relacionar, de una manera sistemática, las calidades térmicas y los costos globales a ellas asociados.

\section{PRIMERA PARTE}

\section{VARIABLES CONSIDERADAS EN EL ANÁLISIS}

Los resultados obtenidos, que se analizarán más adelante, surgen de la comparación de dos o más variables de las que a continuación se mencionan. Estas variables son bioclimáticas, sociales, económicas, tecnológicas y morfológicas.

Desde el punto de vista térmico, este trabajo se ha circunscrito al análisis de dos coeficientes de evaluación del confort térmico: $\mathrm{K}_{\text {y }} \mathrm{K}_{\mathrm{G}}$, cuyas definiciones y formas de cálculo se expresarán más adelante. En la aplicación metodológica que aquí se presenta no fueron considerados el comportamiento higrotérmico y la permeabilidad al aire de los cerramientos; estos aspectos pueden ser incorporados sin dificultad, así como cualquier otro parámetro de medición de las calidades objetivas.

Desde el punto de vista de los costos, se han considerado sólo los valores correspondientes al costo de construcción, de mantenimiento de los items principales del edificio y de la energía necesaria para calefaccionar. No se han tenido en cuenta otros 
aspectos del "costo global" (estudios de factibilidad para la construcción, tierra, honorarios profesionales, impuestos, gastos de administración, etc.). Como en el caso de la calidad, la introducción de estos parámetros no cambia conceptualmente la metodología propuesta, sólo se hace más complejo el cálculo ante el aumento del número de variables.

\section{a) Zonas bioclimáticas de la Provincia de Buenos Aires}

Esta Provincia tiene una superficie de $308.000 \mathrm{~km}^{2}$ (superficie similar a la de Italia), y su población supera los diez millones de habitantes. Se encuentra situada entre los paralelos máximos $33^{\circ}$ y $41^{\circ}$ y los meridianos máximos $56^{\circ}$ y $63^{\circ}$. El territorio presenta características de gran continuidad, ofreciendo el aspecto general de una extensa llanura algo inclinada hacia el oeste. El clima predominante es el templado, con temperaturas medias entre $13^{\circ}$ y $17^{\circ}$.

De acuerdo a la norma IRAM 11.603 (año 1978) de clasificación bioambiental, cuyo objetivo es "zonificar el mapa de la República Argentina según un criterio bioambiental, estableciendo las características térmicas, higrotérmicas y heliofánicas de cada zona"..., la Provincia de Buenos Aires queda dividida en dos zonas climáticas que, a su vez, se subdividen según criterios complementarios (Fig. 2).

La primera zona es la Templada Cálida, que afecta a la mitad norte de la Provincia. Sus amplitudes térmicas son mayores a los $14^{\circ} \mathrm{C}$, excepto al este, donde la cifra es más pequeña. Esta última subárea es comparativamente de poca extensión, pero contiene a la ciudad de Buenos Aires y al conurbano bonaerense, es decir, las zonas con mayor densidad de población.

La segunda zona es la Templada Fría. Se extiende sobre la mitad sur de la Provincia, distinguiéndose al este una angosta franja marítima, y al oeste una zona de transición. Las subáreas de montaña y de máxima irradiancia, también pertenecientes a esta zona bioclimática, quedan fuera de los límites de la Provincia de Buenos Aires.

Además de las zonas bioclimáticas consideradas en la Norma, se tomaron en cuenta otros criterios de evaluación complementarios, como por ejemplo el de las "Regiones ambientales". Este análisis, aparte de las características del medio ambiente físico, incluye datos útiles para ser aplicados al sector de la construcción: deterioro de suelos y de la cobertu-

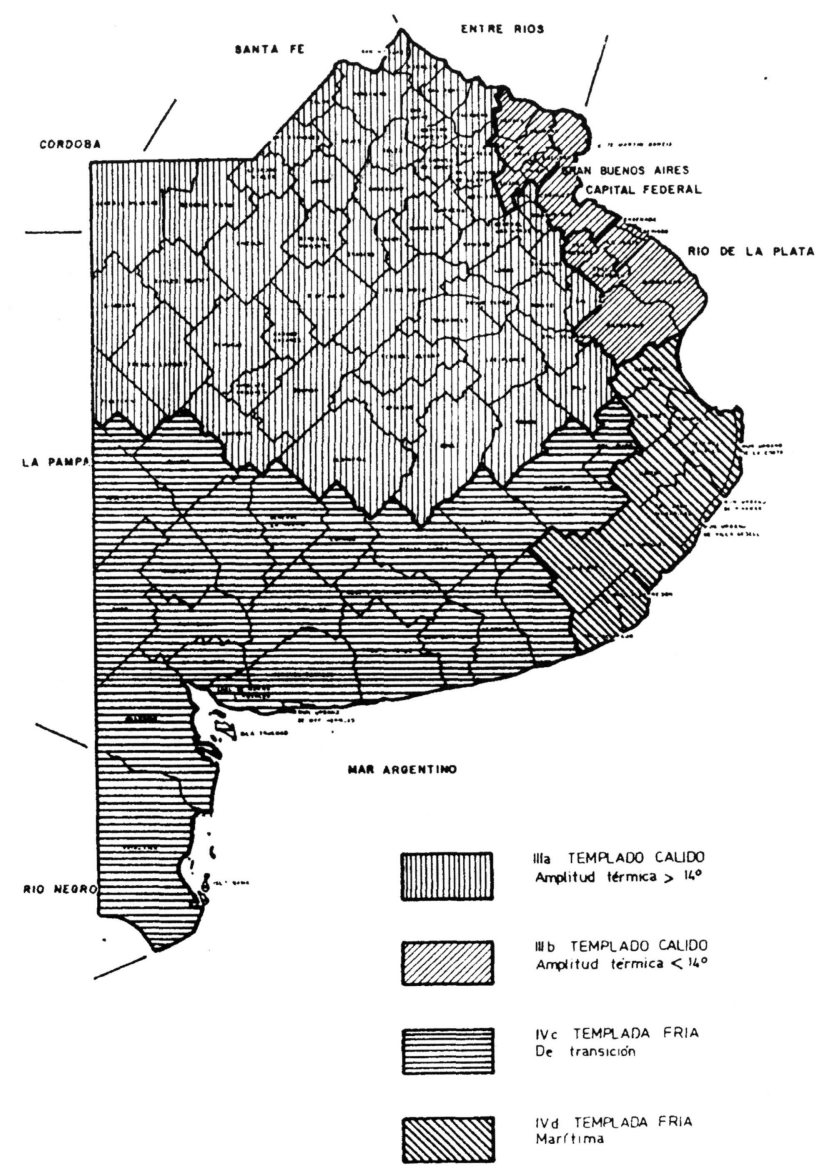

Fig. 2.- Zonas bioambientales.

ra vegetal, reservas mineras, grado de utilización del espacio (medio ambiente urbano y rural), etc. ${ }^{(2)}$.

\section{b) Caracteristicas del entorno - Usuarios representa- tivos}

Es sabido que, en el intercambio térmico de un edificio con el exterior, y en consecuencia en la consideración del ahorro energético, intervienen múltiples factores, aparte de los que han sido mencionados hasta el momento. Por ejemplo:

a) La inercia térmica de paredes y techos.

b) Las ganancias térmicas por asoleamiento e iluminación artificial.

c) Barreras edilicias, vegetales, capaces de modificar los efectos del asoleamiento, ventilación, etc.

d) Los efectos de las "islas calientes" (microclima de ciudades en comparación con las áreas rurales).

e) Las modalidades de uso: calor generado por procesos en el interior de los recintos, variación en el tiempo del número de personas que las habitan, etc. 
En el presente trabajo han sido sólo considerados los puntos d) y e).

En lo que respecta al entorno, se han tenido en cuenta las distintas situaciones típicas en que pueden encontrarse las construcciones, adoptando criterios del CSTB ${ }^{(3)}$ :

- Construcciones situadas en el interior de grandes centros urbanos (la mitad o más de los edificios tienen más de cuatro pisos).

- Construcciones situadas en ciudades pequeñas o medias, o en la periferia de grandes centros urbanos.

- Construcciones aisladas rurales.

- Construcciones aisladas al borde del mar o situadas en ciudades costeras (cuando los edificios estén a una distancia del litoral inferior a 15 veces su altura real, y siempre que las ventanas estén en fachadas no protegidas).

La consideración de las características del usuario como dato clave para el estudio del confort térmico y sus costos tiene, en nuestro medio, escasos antecedentes. Sólo a partir de situaciones coyunturales de crisis energética, los usos y costumbres de los habitantes de las viviendas han comenzado a considerarse, pues de ello depende en gran medida el ahorro energético.

En el caso que nos ocupa, el análisis respecto del usuario se centró en la posible permanencia del mismo dentro de la vivienda. Esta permanencia origina, entre otros efectos, generación de vapor, condensaciones, gasto energético por el uso de artefactos y regulación de las aberturas como moderadoras de la temperatura.

También se tuvieron en cuenta, para trazar el pérfil de los usuarios representativos, datos provenientes de los Censos Nacionales de Población y Vivienda; por ejemplo, el tamaño del hogar, la composición familiar, la inserción ocupacional y el nivel de instrucción ${ }^{(+)}$. Esta información no sólo aporta datos sobre los usos y costumbres de los usuarios, sino también sobre el grado de receptividad que puedan tener campañas para la optimización térmica por medios naturales y el ahorro de energía.

Como puede apreciarse, adoptamos el criterio -tratándose de un trabajo de investigación- de no limitarnos a la aplicación de las Normas de cumplimiento obligatorio, sino de introducir aquellos aspectos que, a nuestro juicio, no han sido considerados en las reglamentaciones sobre el tema.

\section{c) Parámetros de evaluación térmica}

Para la estimación de los parámetros térmicos aplicables a la Provincia de Buenos Aires, se consultaron los valores y métodos de cálculo de las Normas IRAM argentinas, referentes al acondicionamiento térmico de edificios.

A los efectos de una mejor comprensión por parte de los lectores españoles, se empleará la nomenclatura de la Norma Básica NBE-CT-79 sobre condiciones térmicas de los edificios. Se harán comparaciones de valores máximos admisibles para la normativa argentina y española toda vez que ello sea posible. Debe recordarse que entre la Provincia de Buenos Aires y España existen diferencias bioclimáticas, y que las tecnologías de construcción tradicionales utilizadas en la edificación de viviendas presentan variantes.

Las normas argentinas que se emplearon, y que pueden ser consultadas para ampliar dicha comparación, son las siguientes:

- N.I. 11.601: Método de cálculo de la resistividad térmica de muros y techos (1974). (Tabla I).

- N.I. 11.603: Clasificación bioambiental de la República Argentina (1978).

- N.I. 11.604: Condiciones de habitabilidad para invierno en viviendas: coeficiente de transmisión térmica global $\mathrm{K}_{\mathrm{G}}$ (1980). (Tabla II).

- N.I. 11.605: Condiciones de habitabilidad en viviendas, valores máximos admisibles de transmisión térmica K (1980). (Tabla III).

- N.I. 11.625: Verificación del riesgo de condensación de vapor de agua, superficial e intersticial en muros y techos de edificios (1980).

Tabla I.- Conductividad térmica. Comparación entre ambas normativas para algunos ejemplos.

\begin{tabular}{|c|c|c|c|c|c|}
\hline \multirow{2}{*}{$\begin{array}{l}\text { Materialo } \\
\text { elemento }\end{array}$} & \multirow{2}{*}{\begin{tabular}{|c|} 
Densidad \\
aparente \\
$\mathrm{kg} / \mathrm{m}^{*}$
\end{tabular}} & \multicolumn{2}{|c|}{ IRAM 11.601} & \multicolumn{2}{|c|}{ NBE - CT - 79} \\
\hline & & $\mathrm{kcal} / \mathrm{mh}{ }^{\prime} \mathrm{C}$ & $\mathrm{W} / \mathrm{m}^{\circ} \mathrm{C}$ & $\mathrm{kcal} / \mathrm{mh}^{\prime} \mathrm{C}$ & $\mathrm{W} / \mathrm{m} \cdot \mathrm{C}$ \\
\hline $\begin{array}{l}\text { Hormigón con } \\
\text { áridos ligeros }\end{array}$ & 1.000 & 0,31 & 0,36 & 0,28 & 0,33 \\
\hline $\begin{array}{l}\text { Fábrica de } \\
\text { ladrillos macizos }\end{array}$ & 1.800 & 0.78 & 0,91 & 0.75 & 0,87 \\
\hline Vidrio plano & $\begin{array}{l}2.700 \\
2.500\end{array}$ & 0.70 & 0,81 & 0,82 & 0,95 \\
\hline $\begin{array}{l}\text { Mortero de } \\
\text { cemento }\end{array}$ & $\begin{array}{l}2.100 \\
2.000\end{array}$ & 1,20 & 1,40 & 1,20 & 1,40 \\
\hline $\begin{array}{l}\text { Tablero de } \\
\text { partículas } \\
\text { aglomeradas }\end{array}$ & $\begin{array}{l}600 \\
650 \\
\end{array}$ & 0.085 & 0,09 & 0,07 & 0,08 \\
\hline $\begin{array}{l}\text { Enlucidos de } \\
\text { yeso }\end{array}$ & 800 & 0,34 & $0.40^{-}$ & 0,26 & 0.30 \\
\hline
\end{tabular}

Nota: Cuan 
Tabla II.- Valores límite máximos de $K_{G}$ en $k c a l / m^{3} h{ }^{\circ} \mathrm{C}-\left(W / m^{3}{ }^{\circ} \mathbf{C}\right)$

\begin{tabular}{|c|c|c|}
\hline \multirow{2}{*}{$\begin{array}{l}\text { FACTOR IEE } \\
\text { FORMA F } \\
(\mathrm{m}-1)\end{array}$} & \multicolumn{2}{|c|}{ ZONA BIOA.MBIENTAL } \\
\hline & $\begin{array}{c}\text { III } \\
\text { TEMPLADA } \\
\text { CÁLIDA }\end{array}$ & $\begin{array}{c}\text { IV } \\
\text { TEMPLADA } \\
\text { FRIA }\end{array}$ \\
\hline$<0,20$ & $\begin{array}{c}0.66 \\
(0.77)\end{array}$ & $\begin{array}{c}0,60 \\
(0,70)\end{array}$ \\
\hline 0.30 & $\begin{array}{c}0.76 \\
(0.88)\end{array}$ & $\begin{array}{c}0.70 \\
(0.81)\end{array}$ \\
\hline 0.40 & $\begin{array}{c}0.85 \\
(0.99) \\
\end{array}$ & $\begin{array}{c}0.78 \\
(0.91) \\
\end{array}$ \\
\hline 0.50 & $\begin{array}{r}0,97 \\
(1,13) \\
\end{array}$ & $\begin{array}{r}0.67 \\
(1.01) \\
\end{array}$ \\
\hline 0.60 & $\begin{array}{c}1.05 \\
(1.22)\end{array}$ & $\begin{array}{c}0.99) \\
(1.15)\end{array}$ \\
\hline 0,70 & $\begin{array}{c}1.14 \\
(1.33) \\
\end{array}$ & $\begin{array}{c}1,09 \\
(1.27) \\
\end{array}$ \\
\hline 0.80 & $\begin{array}{c}1.26 \\
(1 .+7) \\
\end{array}$ & $\begin{array}{r}1.16 \\
(1.85) \\
\end{array}$ \\
\hline 0.90 & $\begin{array}{r}1.38 \\
(1.60) \\
\end{array}$ & $\begin{array}{r}1.30 \\
(1.51) \\
\end{array}$ \\
\hline 1.00 & $\begin{array}{c}1.51 \\
(1,76) \\
\end{array}$ & $\begin{array}{r}1,40 \\
(1,63) \\
\end{array}$ \\
\hline$>1.20$ & $\begin{array}{r}1,77 \\
(2,06) \\
\end{array}$ & $\begin{array}{r}1.6 .3 \\
(1.90) \\
\end{array}$ \\
\hline
\end{tabular}

\begin{tabular}{|c|c|c|c|c|c|c|}
\hline \multirow{2}{*}{$\begin{array}{l}\text { THOO DE ENERGIA } \\
\text { PARA } \\
\text { CALEFACCION }\end{array}$} & \multirow{2}{*}{$\begin{array}{l}\text { FACTOR DE: } \\
\text { FORMA } \\
(\mathrm{ml}-1)\end{array}$} & \multicolumn{5}{|c|}{ ZONA CLIMÁTICA (SEGÚN MAPA 1 , art. 13\%) } \\
\hline & & A & 13 & C & 1) & $\mathrm{E}$ \\
\hline \multirow{2}{*}{$\begin{array}{l}\text { Combustibles } \\
\text { sólidos, licquiclos } \\
\text { ogatseosos }\end{array}$} & $<0.25$ & $2.10(2.45)$ & $1.61(1.89)$ & $1.40(1.61)$ & $1.26(1,47)$ & $1.19(1 .+0)$ \\
\hline & $>1.00$ & $1,20(1.40)$ & $0.92(1.08)$ & $0.80(0.92)$ & $0.72(0.84)$ & $0.68(0.80)$ \\
\hline \multirow{2}{*}{$\begin{array}{l}\text { Eclificios sin calefacción } \\
\text { ocalefactaclos con } \\
\text { energía } \\
\text { eléctrica directa por } \\
\text { efecto Joule }\end{array}$} & $<0,25$ & $2.10(2 .+5)$ & $1.40(1.61)$ & $1,05(1.19)$ & $0.91(1.05)$ & $0.77(0.91)$ \\
\hline & $>1.00$ & $1,20(1.4(0)$ & $0.80(0,92)$ & $0,60(0.68)$ & $0.52(0.60)$ & $0.45(0.52)$ \\
\hline
\end{tabular}

donde "a" es un coeficiente que se obtiene en la tabla 1 bis, artículo to de la NBE - CT - 79.

La evaluación de las diferentes soluciones habitacionales se realizó a través del coeficiente de transmisión térmica $\mathrm{K}$ y el coeficiente de transmisión térmica global $\mathrm{K}_{\mathrm{G}}$. Recordaremos el significado de ambos términos.

$\mathrm{K}$ (medible en $\mathrm{kcal} / \mathrm{m}^{2} \mathrm{~h}{ }^{\circ} \mathrm{C}$ ó $\mathrm{W} / \mathrm{m}^{2}{ }^{\circ} \mathrm{C}$ ) es el flujo de calor por unidad de superficie y por grado de temperatura entre dos ambientes contiguos. Se parte de la hipótesis que el cerramiento tiene caras isotermas, y que separa dos ambientes también isotermos.

$$
\mathrm{K}=\frac{1}{\frac{1}{\mathrm{he}}+\frac{\mathrm{L} 1}{\lambda 1}+\frac{\mathrm{L} 1}{\lambda 2}+\ldots \frac{1}{\mathrm{hi}}}
$$

donde:

$\frac{1}{\text { he }}$ y $\frac{1}{h i}=\underset{ }{\left(\mathrm{m}^{2} \mathrm{~h}{ }^{\circ} \mathrm{C} / \mathrm{kcal} \text { ó } \mathrm{cm}^{2}{ }^{\circ} \mathrm{C} / \mathrm{W}\right)}$

$\frac{\mathrm{L} 1}{\lambda 1}$ y $\frac{\mathrm{L} 2}{\lambda 2}=$ resistencias parciales de las distintas láminas que pueden componer la pared.

$\mathrm{K}_{\mathrm{G}}$ es el coeficiente de transmisión térmica global de un edificio $\left(\mathrm{kcal} / \mathrm{m}^{3} \mathrm{~h}{ }^{\circ} \mathrm{C}\right.$ ó $\left.\mathrm{W} / \mathrm{m}^{3}{ }^{\circ} \mathrm{C}\right)$, que es la media ponderada de los coeficientes $\mathrm{K}$ de transmisión de calor de los cerramientos que envuelven un edificio. 
Tabla III.- Valores de K máximos admisibles, en $\mathbf{k c a l} / \mathbf{m}^{2} \mathbf{h}{ }^{\circ} \mathrm{C}-\left(\mathrm{W} / \mathbf{m}^{2}{ }^{\circ} \mathrm{C}\right)$

\begin{tabular}{|c|c|c|c|c|}
\hline \multirow{3}{*}{ CERRAMIENTO } & \multirow{3}{*}{ ORIENTACIÓN } & \multicolumn{3}{|c|}{ ZONA BIOAMBIENTAL } \\
\hline & & \multicolumn{2}{|c|}{ III } & \multirow{2}{*}{ IV } \\
\hline & & a & b & \\
\hline Muros & NyS & $1.3+\mathrm{mt}$ & $\begin{array}{c}1.6+\mathrm{mt} \\
(1.86+1.16 \mathrm{mt})\end{array}$ & \multirow{2}{*}{$\begin{array}{c}1.3+\mathrm{mt} \\
(1.51+1,16 \mathrm{mt}\end{array}$} \\
\hline exteriores & E y $\mathrm{O}$ & $(1.51+1.16 \mathrm{mt})$ & $\begin{array}{c}1,3+\mathrm{mt} \\
(1,51+1,16 \mathrm{mt})\end{array}$ & \\
\hline Techos & $\sigma$ & \multicolumn{2}{|c|}{$\begin{array}{c}0,9+\mathrm{mt} \\
(1.04+1,16 \mathrm{mt})\end{array}$} & $\begin{array}{c}0.9+m t \\
(1.04+1.16 \mathrm{mt})\end{array}$ \\
\hline
\end{tabular}

a) Norma IRAM $11.605(1980)$

$\mathrm{mt}$ : masa del cerramiento, expresada en $\mathrm{t} / \mathrm{m}^{-}$

Techos: el valor del $\mathrm{K}$ máximo admisible se calcula para ambos sentidos del flujo de calor

\begin{tabular}{|c|c|c|c|c|c|}
\hline \multirow{2}{*}{\multicolumn{2}{|c|}{ TIPO DE CERRAMIENTO }} & \multicolumn{4}{|c|}{$\begin{array}{c}\text { ZONA CLIMÁTICA } \\
\text { (SEGÚN MAPA 2. art. 13) }\end{array}$} \\
\hline & & V Y W & $\mathrm{x}$ & Y & Z \\
\hline \multirow{4}{*}{ Cerramientos exteriores } & Cubiertas & $\begin{array}{l}1,20 \\
(1,40)\end{array}$ & $\begin{array}{l}1.03 \\
(1.20)\end{array}$ & $\begin{array}{l}0,77 \\
(0,90)\end{array}$ & $\begin{array}{l}0.60 \\
(0.70)\end{array}$ \\
\hline & $\begin{array}{l}\text { Fachadas ligeras } \\
\left(<200 \mathrm{~kg} / \mathrm{m}^{2}\right)\end{array}$ & $\begin{array}{l}1.03 \\
(1.20)\end{array}$ & $\begin{array}{r}1,03 \\
(1,20)\end{array}$ & $\begin{array}{r}1,03 \\
(1,20)\end{array}$ & $\begin{array}{r}1.03 \\
(1.20)\end{array}$ \\
\hline & $\begin{array}{l}\text { Fachatdals pesadlas } \\
\qquad\left(>200 \mathrm{~kg} / \mathrm{m}^{2}\right)\end{array}$ & $\begin{array}{l}1.55 \\
(1.80)\end{array}$ & $\begin{array}{l}1.38 \\
(1,60)\end{array}$ & $\begin{array}{r}1.20 \\
(1.40)\end{array}$ & $\begin{array}{r}1.20 \\
(1.40)\end{array}$ \\
\hline & $\begin{array}{l}\text { Forjados sobre } \\
\text { espacio abierto }\end{array}$ & $\begin{array}{l}0,86 \\
(1,00)\end{array}$ & $\begin{array}{l}0,77 \\
(0,90)\end{array}$ & $\begin{array}{l}0,69 \\
(0,80)\end{array}$ & $\begin{array}{l}0,60 \\
(0,70)\end{array}$ \\
\hline \multirow{2}{*}{$\begin{array}{l}\text { Cerramientos c/locales } \\
\text { no calefaccionados }\end{array}$} & Paredes & $\begin{array}{l}1.72 \\
(2,00)\end{array}$ & $\begin{array}{l}1.55 \\
(1,80)\end{array}$ & $\begin{array}{l}1,38 \\
(1,60)\end{array}$ & $\begin{array}{l}1.38 \\
(1.60)\end{array}$ \\
\hline & Suelos y techos & & $\begin{array}{l}1,20 \\
(1.40)\end{array}$ & $\begin{array}{l}1.03 \\
(1,20)\end{array}$ & $\begin{array}{l}1.03 \\
(1.20)\end{array}$ \\
\hline
\end{tabular}

b) NBE - CT - 79

\section{d) Parámetros de evaluación económica}

En el análisis que nos ocupa, se han considerado sólo aquellos costos que parecen relacionarse directamente con la obtención del confort térmico y el ahorro energético:

a) Costo de construcción: comprende el costo de los materiales y de la mano de obra para los cerramientos estudiados. Los valores fueron extraídos de las publicaciones mensuales especializadas para el mes y año en que se realizó el análisis. b) Costo de mantenimiento: se utilizó el método propuesto por J.L. Mascaro, que consiste en estimar costos como porcentajes del costo de construcción a lo largo de la vida útil, para los principales rubros constructivos de la envolvente (techos, muros, revestimientos y carpinterías) $)^{(5)}$.

c) Costo de energía para calefacción: se estimó en base al método más usual de calefactar viviendas en la República Argentina (estufas individuales de tiro balanceado, de $3.200 \mathrm{kcal}$, alimentadas a gas natural, y con una pérdida por chimenea del orden del 20\%). 
Las necesidades de calefacción se calcularon en base a la fórmula:

$\mathrm{Q}=\Delta \mathrm{t} \cdot \mathrm{V}\left(0,7+\mathrm{K}_{\mathrm{G}}\right)($ Norma IRAM 11.604)

donde:

$\mathrm{Q}=$ necesidades de calefacción, en kcal

$\mathrm{V}=$ volumen interior, en $\mathrm{m}^{3}$

$\Delta t=$ diferencia entre la temperatura de diseño interior y exterior (mínimo)

$\mathrm{K}_{\mathrm{G}}=$ coeficiente de transmisión térmica global, en $\mathrm{kcal} / \mathrm{m}^{3} \mathrm{~h}{ }^{\circ} \mathrm{C}$.

A los valores obtenidos se les aplicó el precio del $\mathrm{m}^{3}$ de gas natural, integrado por su costo básico más los impuestos municipales, provinciales y nacionales que determinan su valor definitivo.

\section{e) Tipologias de edificios}

Para este análisis de la relación confort térmico-costos, se eligieron diez conjuntos habitacionales ubicados en la Provincia de Buenos Aires. La elección de los mismos se realizó en base a su variedad, desde aquellos puntos de vista que interesan a la economía y a los aspectos térmicos. Así, se buscaron distintas situaciones en lo que respecta a:

- Morfología y tipología de edificios y viviendas.

- Ubicación de la vivienda dentro del edificio colectivo.

- Grado de compacidad.

- Ubicación en diferentes zonas bioclimáticas.

- Tecnología empleada en la envolvente.

Ningún ejemplo supera la altura de planta baja y tres pisos altos. Por lo tanto, no se han considerado parámetros térmicos diferenciales con la variación de la altura, como sería el caso de la presión del viento.

\section{SEGUNDA PARTE}

\section{ESTUDIO TÉRMICO: ECONÓMICO PARA VIVIEN-} DAS DE DOS DORMITORIOS

En base a la información reunida se combinaron, con el apoyo de programas de computación, los valores hallados para las variables de costo y calidades térmicas. El objetivo fue establecer un orden de preferencia, para observar cuándo se optimiza la mencionada relación costo-calidad, considerando

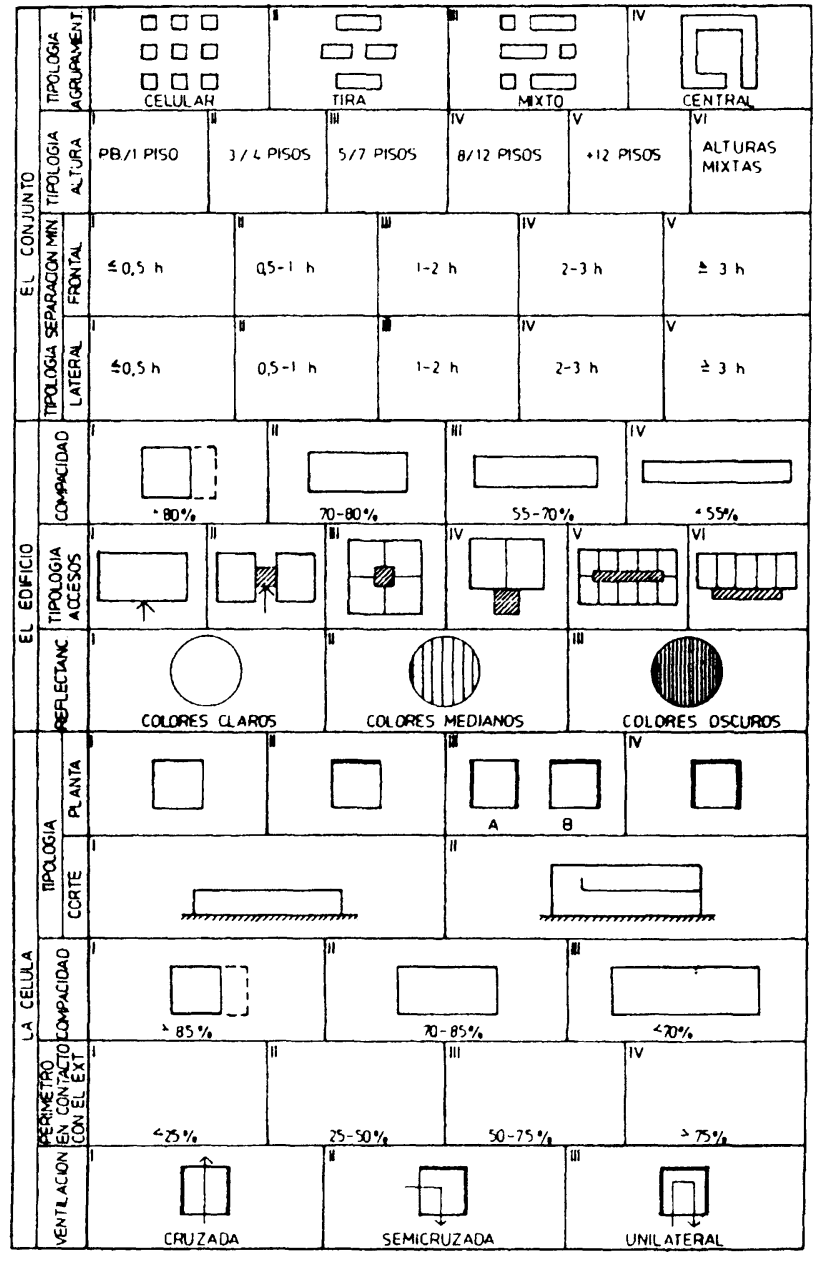

Fig. 3.- Sistema de códigos de identificación.

posibilidades alternativas morfológicas y tecnológicas, para la envolvente de los edificios. También se incluyó como variable la ubicación de las viviendas en el edificio colectivo (entre medianeras, en el extremo de un bloque, en el último piso, etc.).

A los efectos comparativos, se utilizaron seis tipos de muros, donde se varía el tipo y espesor de los mampuestos, y se mantienen constantes sus revestimientos (revoque exterior de mortero de cemento, mortero y enlucido a la cal; e interior de mortero y enlucido de cal). También se mantuvieron constantes el tamaño y tipo de carpinterías (marco y hojas metálicas, ventanas corredizas, vidrio plano), y la cubierta (losa plana de hormigón armado sin aislamiento térmico). Hay que destacar que las tecnologías elegidas son las más habituales en la República Argentina en edificios colectivos para usuarios de recursos limitados.

No se analizaron tampoco las propiedades estructurales de los muros y su significado éconómico (mayores costos de soporte según el peso de los muros, etc.). 
Tabla IV

\begin{tabular}{|c|c|c|c|c|}
\hline ESCALA & TTEM & & CÓnlGO & CARACTERISTICAS \\
\hline \multirow{5}{*}{ 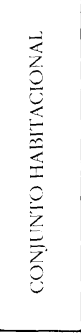 } & \multicolumn{2}{|c|}{ Nombre } & $\mathrm{A} 1$ & Leandro $N$ Alen \\
\hline & \multicolumn{2}{|c|}{ Clima/entorno } & 2012 & $\begin{array}{l}\text { Zona templada cálida Amplitud térmica } \\
\text { menor a } 1+\mathrm{C} / \text { Region metropolitana } \\
\text { Cindades pequenaso medias o periferia de } \\
\text { grandles centros urbanos Mayore's a } 30.000 \\
\text { habitintes }\end{array}$ \\
\hline & \multicolumn{2}{|l|}{ Alturat } & VI & Alturas mixtas \\
\hline & \multirow{2}{*}{ 垈 } & Frontal & II & $1 / 2$ de lia altura del edificio \\
\hline & & Lateral & II & $1 / 2$ de la altura del edificio \\
\hline \multirow{4}{*}{ 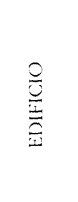 } & \multicolumn{2}{|c|}{ Tipologia accesos } & $\mathrm{VT}$ & Palier externo \\
\hline & \multicolumn{2}{|c|}{ Área $\left(\mathrm{m}^{2}\right)$} & - & 860 \\
\hline & \multicolumn{2}{|c|}{ Perímetro (m) } & - & 212 \\
\hline & \multicolumn{2}{|c|}{ Compacidad (nin) } & - & 49 \\
\hline \multirow{8}{*}{ 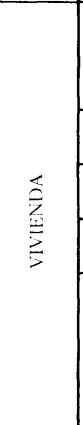 } & \multirow{2}{*}{ 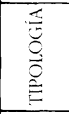 } & Planta & II & 3 lados exentos \\
\hline & & Corte & II & En contacto con cubierta. extremo en plantit \\
\hline & \multicolumn{2}{|c|}{ Compacidad $\%(n)$} & - & 87 \\
\hline & \multicolumn{2}{|c|}{ Perímetro total (m) } & - & 33 \\
\hline & \multicolumn{2}{|c|}{ Perímetro exento (m) } & - & 15 \\
\hline & \multirow{3}{*}{ 恴 } & Exenta $\left(\mathrm{m}^{2}\right)$ & - & 42 \\
\hline & & Muros (\%) & - & 84 \\
\hline & & Vinos $(\%)$ & - & 16 \\
\hline
\end{tabular}

Tabla V

\begin{tabular}{|c|c|c|c|c|c|c|c|c|c|c|c|c|c|}
\hline \multirow{3}{*}{$\begin{array}{l}\text { TIPO DE } \\
\text { MURO }\end{array}$} & \multicolumn{12}{|c|}{ ENVOLVENTE DE LA VIVIENDA } & \multirow{3}{*}{ 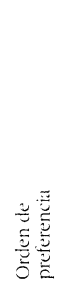 } \\
\hline & \multicolumn{3}{|c|}{$\mathrm{K}(\mathrm{kcal} / \mathrm{h} \mathrm{m} \cdot \mathrm{C})$} & \multicolumn{3}{|c|}{$\mathrm{K}_{\mathrm{G}_{\mathrm{r}}}\left(\mathrm{kcall} / \mathrm{h} \mathrm{m}^{2} \mathrm{C}\right)$} & \multicolumn{4}{|c|}{ COSTOS (AUSTRALES) } & \multicolumn{2}{|c|}{$\begin{array}{c}\text { COSTOS } \\
\text { PONDERADOS }\end{array}$} & \\
\hline & $\stackrel{\Xi}{\underline{E}}$ & 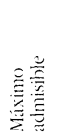 & 旁 & 兯 & 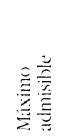 & . & 离 & 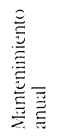 & 莺 & 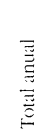 & 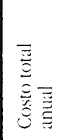 & 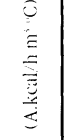 & \\
\hline $\begin{array}{l}\text { Ladrillo común } \\
\quad \because: 0,15 \mathrm{~m}\end{array}$ & 2.56 & 1.62 & NO & $1.2+$ & 1.03 & NO & 2.444 & 50 & 56 & 97 & 120 & & - \\
\hline $\begin{array}{l}\text { Ladrillo común } \\
\text { e: } 0.30 \mathrm{~m}\end{array}$ & 1.75 & 1.87 & sí & 1.03 & 1.03 & Sí & 2.671 & 50 & 54 & 101 & 104 & & 4 \\
\hline $\begin{array}{l}\text { Ladrillo hueco } \\
\text { e: } 0.15 \mathrm{~m}\end{array}$ & 1.59 & 1.41 & NO & 1.05 & 1.03 & NO & 2.458 & 50 & 54 & 96 & 100. & & - \\
\hline $\begin{array}{l}\text { Ladrillo hueco } \\
\text { e: } 0.20 \mathrm{~m}\end{array}$ & 1.30 & 1.63 & sí & 1.00 & 1.03 & sí & 2.534 & 50 & 54 & 98 & 98,0 & & 3 \\
\hline $\begin{array}{l}\text { Bloque cerámico } \\
\text { e: } 0,15 \mathrm{~m}\end{array}$ & 1.16 & 1.53 & sí & 0.97 & 1.03 & sí & 2.371 & 49 & $5+$ & 94 & 91.1 & & 2 \\
\hline $\begin{array}{c}\text { Bloque cerámico } \\
\text { e: } 0.20 \mathrm{~m}\end{array}$ & 0.99 & 1,55 & Sí & 0.94 & 1.03 & sí & 2.387 & 49 & 54 & $9+$ & 88.3 & & 1 \\
\hline
\end{tabular}

NOTA: Para establecer el orden de preferencia del costo ponderadose han considerado sólo los ejemplos que cumplían con los valores máximos alceptados de $\mathrm{K}_{\mathrm{G}}$.

Cada uno de los ejemplos analizados queda definido por un par de Tablas. En la primera (Tabla IV) se anotan los datos geométricos y pautas de diseño en general que caracterizan al conjunto habitacional, al edificio (cuando se trata de viviendas colectivas), y a la célula destinada a vivienda. Para ello se creó previamente un sistema de código de identificación de las distintas posibilidades halladas
(Fig. 3). En la segunda (Tabla V), para seis tipos de muros alternativos, se observan los datos obtenidos al analizar cada ejemplo desde el punto de vista térmico y de costos. Se llega a la estimación de los "costos ponderados" (costos calificados a partir de las aptitudes térmicas del ejemplo estudiado), y a establecer un orden de preferencia de costo y calidad. 
TablaVI.- Tipo de muros: bloque cerámico portante, e: $0,20 \mathrm{~m}$

\begin{tabular}{|c|c|c|c|c|c|c|c|c|c|c|c|}
\hline \multirow[b]{3}{*}{ 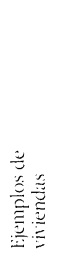 } & \multicolumn{4}{|c|}{ CARACTERÍSTICAS IDE LA VIVIENIAA } & \multirow{3}{*}{ 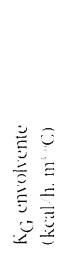 } & \multirow[b]{3}{*}{ } & \multicolumn{3}{|c|}{ COSTOS (australes) } & \multirow{3}{*}{ 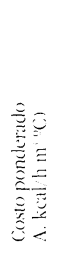 } & \multirow[b]{3}{*}{ 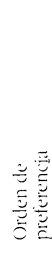 } \\
\hline & \multirow{2}{*}{ 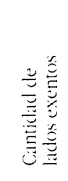 } & \multirow{2}{*}{ 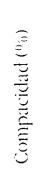 } & \multicolumn{2}{|c|}{ FACHADA EXENTA } & & & \multirow{2}{*}{ 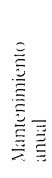 } & \multirow[b]{2}{*}{ 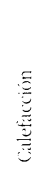 } & \multirow[b]{2}{*}{ 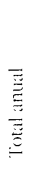 } & & \\
\hline & & & 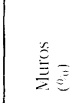 & 咅 & & & & & & & \\
\hline A & $2=$ & 87 & 90 & 10 & 0.68 & 1.711 & 20 & +1 & 62 & +2.16 & 3 \\
\hline 13 & 1 & 76 & 87 & 13 & 0.61 & 1.182 & 18 & 39) & 56 & $3+16$ & 2 \\
\hline C & $2=$ & 88 & 79 & 21 & 0.70 & 1.628 & 23 & 39 & 61 & +2.70 & 5 \\
\hline D) & 2 & 86 & 82 & 18 & 0.76 & 1.460 & 20) & 39 & 56 & +2.56 & + \\
\hline $\mathrm{E}$ & $2=$ & 8() & 85 & 15 & 0.77 & 1.818 & 25 & $H$ & 68 & 52.36 & 6 \\
\hline $\mathrm{F}$ & 2 & 84 & 83 & 17 & $0.6+$ & 1.235 & 17 & 29) & 46 & $29,+4$ & 1 \\
\hline & & & & & & & & & media & +0.50 & \\
\hline
\end{tabular}

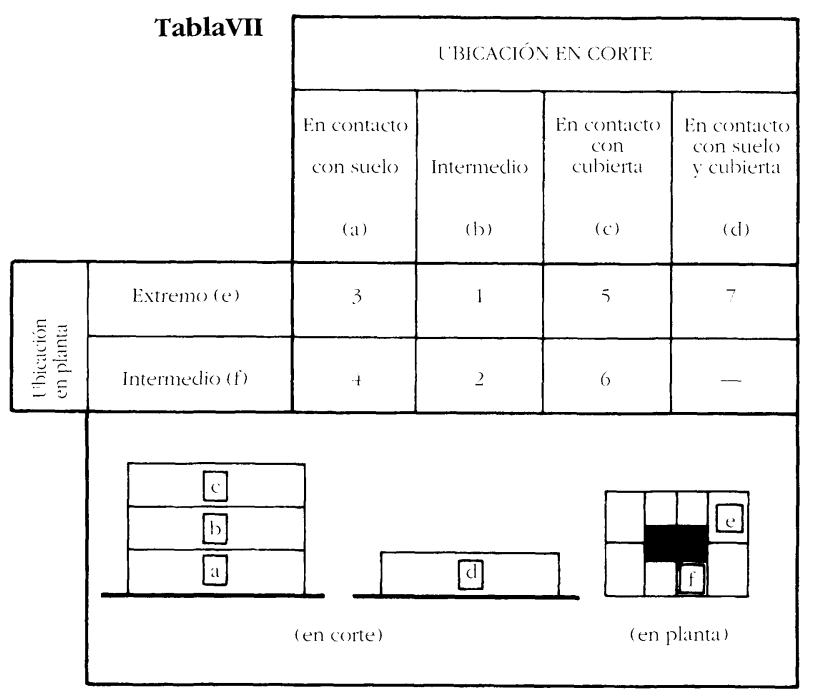

En la Tabla IV sólo cabría especificar qué se entiende por "compacidad". El índice de compacidad se obtiene por comparación entre el perímetro de la vivienda estudiada, y el que tendría si, con la misma superficie, dicho perímetro fuera una circunferencia.

$$
\text { Ic }=\frac{\text { Perímetro de proyecto }}{\text { Perímetro del círculo equivalente }}
$$

A los efectos prácticos, y para evitar el cálculo del denominador de la fórmula, se ha utilizado la siguiente expresión:

$$
=\frac{2 \sqrt{\pi \text {. área de proyecto }}}{\text { Perímetro de proyecto. }}
$$

Los valores de costo, así como los de $\mathrm{K}_{\mathrm{G}}$, son mejores cuanto menor sea su valor. De allí que la ponderación se realice mediante una operación de multiplicación (costo $\mathrm{x} \mathrm{K}_{\mathrm{G}}$ ). El orden de preferencia 1 indica el ejemplo donde se optimiza la relación costo-transmisión térmica global. La conveniencia de la alternativa disminuye a medida que nos alejamos de dicho valor.

A continuación se estudió comparativamente, para cada tipo de muro, todas aquellas viviendas que se encontraban en similar ubicación dentro del edificio colectivo. Como puede observarse, las viviendas difieren en sus características y aun en su superficie, pero todas tienen los mismos requerimientos funcionales (unidades de dos dormitorios, para familias tipo de cuatro personas). 
TablaVIII.- Costos ponderados (australes, $\mathrm{kcal} / \mathrm{h} \mathrm{m}^{3}{ }^{\circ} \mathrm{C}$ )

\begin{tabular}{|c|c|c|c|c|c|c|c|c|}
\hline \multirow[b]{2}{*}{ TIPO DE MURO } & \multicolumn{8}{|c|}{ UBICACIÓN EN EL EDIFICIO } \\
\hline & $\mathrm{a} / \mathrm{e}$ & $a / f$ & $b / e$ & $b / f$ & $\mathrm{c} / \mathrm{e}$ & $\mathrm{c} / \mathrm{f}$ & $\mathrm{d} / \mathrm{e}$ & $d / f$ \\
\hline $\begin{array}{l}\text { Ladrillo común } \\
\quad(\mathrm{e}: 0,15)\end{array}$ & 65,44 & 72,65 & 41,02 & 47,72 & 143,16 & 150,59 & 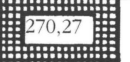 & \multirow{7}{*}{ 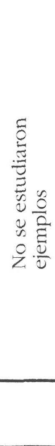 } \\
\hline $\begin{array}{l}\text { Ladrillo común } \\
\quad(\mathrm{e}: 0,30)\end{array}$ & 56,48 & 62,79 & 32,93 & 39,09 & 127,95 & 134,78 & 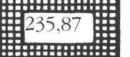 & \\
\hline $\begin{array}{l}\text { Ladrillo hueco } \\
\text { (e: } 0,15)\end{array}$ & 48,77 & 53,20 & 27,52 & 32,70 & 115,51 & 122,69 & 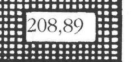 & \\
\hline $\begin{array}{l}\text { Ladrillo hueco } \\
\text { (e: } 0,20)\end{array}$ & 45,65 & 49,31 & 25,17 & 29,61 & 110,48 & 117,23 & 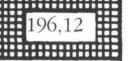 & \\
\hline $\begin{array}{c}\text { Bloque cerámico } \\
\text { (e: } 0,15)\end{array}$ & 39,70 & 43,06 & 21,19 & 24,41 & 100,97 & 107,51 & 171,53 & \\
\hline $\begin{array}{l}\text { Bloque cerámico } \\
\text { (e: } 0,20)\end{array}$ & 37,03 & 40,56 & 19,94 & 22,70 & 97,88 & 103,31 & 164,22 & \\
\hline $\begin{array}{c}\text { ORDEN DE } \\
\text { PREFERENCIA }\end{array}$ & 3 & 4 & 1 & 2 & 5 & 6 & 7 & \\
\hline
\end{tabular}

Valores menores

\begin{tabular}{|c|c|c|c|c|c|c|}
\hline \multirow[t]{2}{*}{ Tabla.- IX } & \multicolumn{2}{|c|}{ URBANO } & \multicolumn{2}{|c|}{ RURAL } & \multicolumn{2}{|c|}{ TOTAL } \\
\hline & № & $\%$ & № & $\%$ & № & $\%$ \\
\hline $\begin{array}{c}\text { Templado cálido } \\
\text { A.T. }>14^{\circ} \mathrm{C}\end{array}$ & 63.139 & 11 & 40.510 & 53 & 103.649 & 16 \\
\hline $\begin{array}{c}\text { Templado cálido } \\
\text { A.T. }<14^{\circ} \mathrm{C} \\
\end{array}$ & 473.834 & 81 & 17.235 & 23 & 491.069 & 74 \\
\hline $\begin{array}{l}\text { Templado frío } \\
\text { de transición }\end{array}$ & 24.489 & 4 & 12.673 & 17 & 37.171 & 6 \\
\hline Templado frío marítimo & 24.240 & 4 & 5.423 & 7 & 29.663 & 4 \\
\hline TOTAL: & 585.711 & 100 & 75.841 & 100 & 661.552 & 100 \\
\hline & \multicolumn{3}{|c|}{$\begin{array}{r}\text { Total } 4 \text { zonas: } \\
\text { Urbano: } \\
\text { Rural: }\end{array}$} & $\begin{array}{l}552 \\
7711 \\
841\end{array}$ & \multicolumn{2}{|c|}{$\begin{array}{r}(100 \%) \\
(89 \%) \\
(11 \%)\end{array}$} \\
\hline
\end{tabular}

En la Tabla VI se muestra el análisis para un ejemplo: seis tipos diferentes de viviendas, todas resueltas con el mismo tipo de envolvente vertical (bloque cerámico de 0,20 metros de espesor), y todas con igual ubicación en el edificio respecto del exterior (en contacto con el suelo, intermedias en planta baja; tipo a/f en la Tabla VII).

Si se calculan los valores de costo ponderado medio para todas las viviendas y tipos de muros, y se los pone en función de cada tipo de ubicación en el edificio, se obtiene la Tabla VIII. Allí puede observarse que, de acuerdo a las coordenadas espacio-temporales consideradas (Provincia de Buenos Aires, octubre de 1985), a las morfologías y tecnologías constructivas tomadas como muestra, y a la normativa de aplicación para dicha zona, se obtienen costos ponderados que varían entre $20 \mathrm{y}$ $270 \mathrm{Ax} \mathrm{kcal} / \mathrm{m}^{3} \mathrm{~h}{ }^{\circ} \mathrm{C}$. Esta variación de más de $1.000 \%$, insistimos, corresponde a soluciones desti- nadas a satisfacer los mismos requisitos funcionales.

Los valores de costo ponderado que se citan en las tablas V, VI y VIII están medidos en australes $\mathrm{x}$ $\mathrm{kcal} / \mathrm{h} \mathrm{m}^{3}{ }^{\circ} \mathrm{C}$. Los costos corresponden a valores de octubre de 1985 (un austral = 1,07 dólares).

Puede apreciarse que, de acuerdo a estos datos, se ha construido un número masivo de viviendas cuyos aspectos morfológicos y tecnológicos no respondían a las características bioclimáticas de cada zona. Por su parte, se da la paradoja que a veces costos iniciales altos no corresponden a las mejores respuestas desde el punto de vista térmico. Esta situación, además, incrementa los costos de operación del edificio, especialmente en lo que se refiere a la energía para calefacción. Todo ello conduce a una falta de optimización de la relación entre el costo total y los aspectos de calidad analizados. 
Informes de la Construcción, Vol. 45 n" 429, enero/febrero 1994

\section{CONCLUSIONES}

Si se tienen en cuenta la totalidad de las variables estudiadas, es posible llegar a conclusiones de carácter general, particular y específico. Comentaremos algunas que, a nuestro juicio, revelan particular interés.

Del déficit habitacional total de la Provincia de Buenos Aires, aproximadamente un 75\% corresponde a la Zona Templada Cálida con amplitud térmica menor a los $14^{\circ} \mathrm{C}$. Dentro de dicha zona, el $80 \%$ del déficit es de tipo urbano. La mayor carencia habitacional se encuentra en la zona bioambiental de menor superficie (Tabla IX).

Este área se caracteriza por inviernos relativamente benignos, y veranos algo calurosos, con tensiones de vapor bajas durante todo el año. Las normas de habitabilidad sugieren para esta zona evitar la incidencia exagerada de la radiación solar en verano, y la adecuada protección de las aberturas. Se recomienda evitar la orientación oeste, siendo los rumbos favorables los que van del NO al E en latitudes superiores a los $30^{\circ}$, y del $\mathrm{NO}$ al SE en las inferiores a $\operatorname{los} 30^{\circ}$ (hemisferio sur).

Estas pautas cobran especial importancia, pues deben regir el diseño de las casi quinientas mil viviendas que conforman el déficit habitacional más crítico de la Provincia.

En lo que se refiere al costo ponderado de las viviendas, los valores medios menores correspondieron a la opción que tiene muros construidos con bloques cerámicos portantes. Se trata de bloques huecos de cerámica roja, con concentración de material en los bordes para aumentar el momento de inercia de la sección transversal, con aprovechamiento máximo de la resistencia del ladrillo. Los hay comunes y pesados. Según su peso y espesor, el coeficiente $\mathrm{K}$ varía entre 0,99 y $1,61 \mathrm{kcal} / \mathrm{m}^{2} \mathrm{~h}{ }^{\circ} \mathrm{C}$.
Desde el punto de vista de la ubicación dentro de un edificio colectivo, la relación costo-confort térmico se minimiza cuando la vivienda está situada en niveles intermedios (no en planta baja o en el último piso); esto es lógico, ya que los forjados interiores son más económicos que los de piso o techo, y porque sólo existen a través de ellos pérdidas o ganancias térmicas de menor incidencia.

El orden de preferencia obtenido es altamente dinámico, y varía al modificarse en el tiempo el costo relativo de cada alternativa. De allí la importancia de contar con un programa alimentable con los datos actualizados de costo.

En general, el análisis realizado demostró que para lograr un confort térmico adecuado no es imprescindible optar por tecnologías costosas. Se da el caso -como en el ejemplo del bloque cerámico portante-que un mampuesto es a la vez más económico y el que tiene menor transmisión térmica entre varias alternativas.

Distintos estudios han demostrado que los problemas que se presentan en los edificios en uso provienen en gran medida de decisiones erróneas tomadas durante el proceso de diseño(6). Cuando luego se quieren solucionar, en forma por lo menos parcial, las manifestaciones patológicas -como los defectos de habitabilidad- se originan exagerados costos de mantenimiento y operación de los edificios.

La transmisión térmica global moderada, el control de las pérdidas y ganancias energéticas, la ventilación natural como factor de regulación climático, no se logran en muchos casos elevando el presupuesto inicial de costos. La relación costo-calidad puede ser optimizada si estas dos variables clave tienen un papel protagonista. 
(1). Amarilla, B.C.: "Costos del confort térmico en viviendas de interés social en la Provincia de Buenos Aires". Comisión de Investigaciones Científicas de la Provincia de Buenos Aires, La Plata, 1986.

(2). Instituto de la Vivienda de la Provincia de Buenos Aires/Departamento Programación: "Metodología general para programar y prioritar conjuntos habitacionales" Documento de avance II. La Plata, 1984.

(3). Centre Scientifique et Technique du Bâtiment (CSTB): "Règles de calcul du coefficient $\mathrm{G}$ des logements et d'autres locaux d’habitation". Paris, 1984
(4). Instituto Nacional de Estadísticas y Censos (INDEC): "Censo de Población y Vivienda 1980".

(5). Mascaró, J.L.: "Métodos de evaluación de proyectos". Facultad de Arquitectura y Urbanismo, Universidad Nacional de La Plata, 1979 .

(6). Comité Euro-internacional del Hormigón - Boletín 157 del CEB: "Control de calidad y garantía de calidad para estructuras de hormigón". Asociación española para el control de calidad, Madrid, 1983.

\section{publicaciones del ICCET/CSIC}

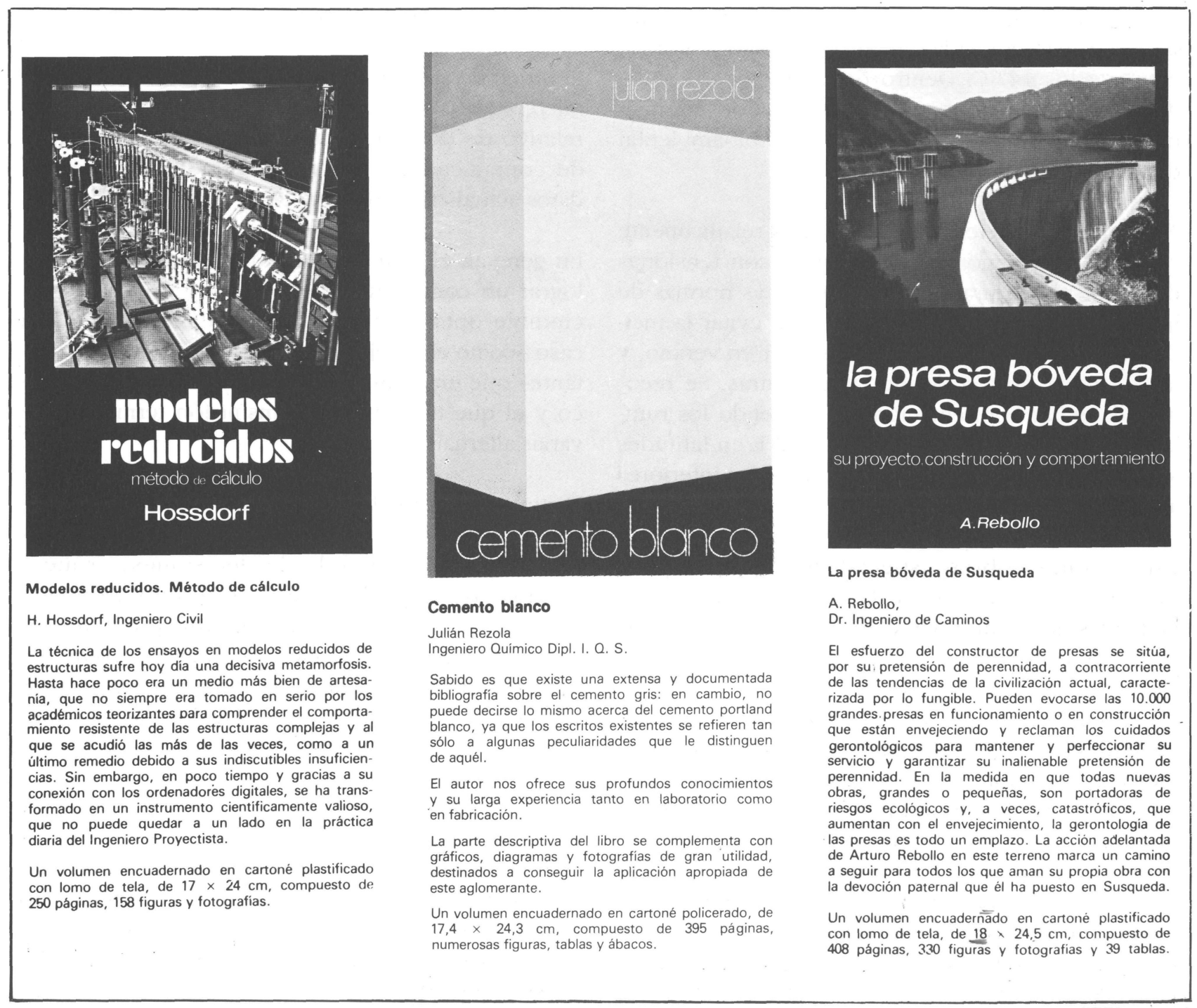

